\title{
Clostridium difficile Colitis, Treatment and Management
}

\author{
Robert Hasty • Vincenzo Barbato $\cdot$ Pedro Valdes • \\ Christopher Sitler
}

Published online: 7 May 2013

(C) Springer Science+Business Media New York 2013

\begin{abstract}
Clostridium difficile is a gram positive, anaerobic, spore forming bacilli, which is an ever-present danger within hospitalized patients accounting for $\sim 15,000$ deaths each year in the United States CDC (MMWR 61:157-162, 2012). Clostridium difficile infection is generally defined by the presence of diarrhea, leukocytosis and testing positive for the organism's toxin via toxin assay. As with most bacterial organisms that developed resistance to antibiotics, the organism has developed several fluoroquinolone resistant strains, BI, NAP1, and toxinotype III and ribotype 027 Warney et al. (Lancet 366:1079-1084, 2005). Not only do we see this bacterium with acute and chronic disruption of the intestinal flora, but it has also been associated with chronic proton pump inhibitor usage and elevation of gastric $\mathrm{pH}$ Howell et al. (Arch Intern Med 170:784-790, 2010••). Proper hand sanitation and contact precautions are two of the most effective methods in preventing the spread of spores. Treatment modalities for this infection have steadily progressed to include metronidazole, vancomycin, oral administration of IV vancomycin, fidaxomicin, and lastly fecal transplantation. Of greatest importance remains our ability to properly diagnose, treat, and prevent this costly and dangerous infection.
\end{abstract}

R. Hasty ( $\square)$

Campbell University School of Osteopathic Medicine,

Buies Creek, NC, USA

e-mail: rhasty@me.com

V. Barbato $\cdot$ P. Valdes

Internal Medicine Residency, Nova Southeastern University College of Osteopathic Medicine/Palmetto General Hospital, Hialeah, FL, USA

C. Sitler

Piedmont Newnan Hospital, Newnan, Georgia, USA
Keywords Clostridium difficile - Fecal bacteriotherapy · Toxic megacolon · Pseudomembranous colitis - Recurrent Clostridium difficile · Probiotics · Fidaxomicin .

Vancomycin taper

\section{Introduction}

Clostridium difficile colitis has become an important clinical entity and currently affects 13 in 1,000 inpatients and accounts for 7,178 inpatients on any given day in American healthcare institutions [11]. There are $\sim 15,000$ deaths each year in the United States which is about the same number of deaths attributable to abdominal aortic aneurysms [1]. The incidence of this entity has increased from 31 cases per 100,000 population in 1996-1984 cases per 100,000 population in 2005 [3]. Clostridium difficile has also dramatically grown in scope in terms of populations affected. For instance, there has been a 12-fold increased risk among children [12]. This article will discuss the reasons for the increase of the disease, current and emerging diagnostic and treatment strategies, as well as methods to prevent $C$. difficile infection.

\section{Definition}

Clostridium difficile infection (CDI) is defined by the presence of symptoms (usually diarrhea) plus a stool test positive for $C$. difficile toxins or colonoscopic and histopathologic findings revealing pseudomembranous colitis.

\section{The New Strain}

Over the past ten years, a more virulent strain of $C$. difficile has emerged. The new strain has been referred to by a number 
of different designations, such as BI, NAP1, toxinotype III, and ribotype 027 , based upon the technology used to isolate or identify the species. This new strain produces 16-23 times more toxins A and B in vitro than do other strains. This new species is also fluoroquinolone resistant. These two features seem to contribute to the increase in morbidity and mortality that we have seen clinically [20].

\section{Proton Pump Inhibitors and Clostridium difficile}

Proton pump inhibitors (PPI) work by increasing the stomach $\mathrm{pH}$. This change in the gastrointestinal acidity alters the bodies innate defense mechanism against pathogenic organisms. Data suggests that there is a four-fold increase risk of $C$. difficile in hospitalized patients who are administered a PPI [9*0]. Additionally, PPI's have been shown to increase recurrence of $C$. difficile by $42 \%$ [9*0]. It is prudent for physicians to balance these risks with the potential benefit when deciding whether to prescribe PPI therapy to a hospitalized patient [14].

Possible reasons for increased incidence of Clostridium difficile colitis

New strain

Increase in antibiotic use

Widespread use of proton pump inhibitors

Predominant use of alcohol-based hand sanitizers

\section{Hand Hygiene}

A recent study demonstrated that $\sim 95 \%$ of all cases of CDI are due to healthcare exposure [1]. The Centers for Disease Control published a set of guidelines in 2002 that recommends clinicians use alcohol-based hand rubs for hand hygiene in most circumstances [2]. The spores of C. difficile are resistant to these alcohol-based hand rubs and the spores can easily be transferred by touch, including hand shaking after use of the alcohol-based hand rubs. Healthcare providers should wash their hands thoroughly with soap and water when caring for patients with CDI to decrease the chance of transmission [10].

\section{Diagnostic Testing}

In general, only patients with diarrhea should be tested for C. difficile. The exception would be if an ileus is present. Testing asymptomatic patients should be avoided, including patients with recent infection (e.g., no "test of cure"). Fecal leukocytes continued to be useful in patients with suspected CDI. They are present in about one-half of all patients with
CDI. Stool culture is the gold standard, but is not practical clinically due to the time it takes to grow the culture. Enzyme immunoassay is a rapid test, but it is suboptimal to the cell cytotoxin assay. Toxin testing is the most important test clinically, but it is less sensitive than other tests. Some sites are now using a 2-step method where glutamate dehydrogenase enzyme immunoassay is performed if the toxin testing is positive. Polymerase chain reaction testing is a rapid, sensitive, and specific test, but more clinical evidence is needed before it is routinely recommended. Repeat testing during the same period of diarrhea is of limited value and should be discouraged [4••].

\section{Unexplained Leukocytosis}

Leukocytosis is common in patients with CDI. One study demonstrated that $58 \%$ of all patients with unexplained leukocytosis had $C$. difficile present [19]. Clinicians should be mindful of this association and consider CDI when caring for a hospitalized patient with unexplained leukocytosis.

\section{Toxic Megacolon}

Toxic megacolon is a potentially lethal complication of CDI. The incidence is $<5 \%$. Early surgical interventions has been shown to decrease the mortality from 22 to $1.2 \%$ $[6,7,18]$. It is important for clinicians to recognize patients with toxic megacolon in order to refer appropriately. Toxic megacolon is defined as radiographic evidence of colonic distention PLUS at least three of the following:

(1) Fever $>38^{\circ} \mathrm{C}$

(2) Heart Rate $>120$ beats/min

(3) Neutrophilic Leukocytosis $>10,500 / \mu \mathrm{L}$

(4) Anemia

In addition, one of the following must be present as well:

(1) Dehydration

(2) Altered sensorium

(3) Electrolyte disturbances

(4) Hypotension [17]

Consider subtotal colectomy with preservation of rectum as the surgical procedure of choice in a patient with a toxic megacolon. Colonoscopies should be avoided in patients with a suspected toxic megacolon due to potential for perforation of the affected bowel. Antiperistaltic agents should be avoided in patients with or suspected CDI due to risk of precipitating toxic megacolon or obscuring symptoms. Bile acid resins, such as cholestyramine would be preferential agents for symptomatic relief and are not associated with toxic megacolon $[4 \bullet \cdot]$. 


\section{Treatment}

It is important to discontinue the inciting antimicrobial as soon as possible when a patient is diagnosed with CDI. When severe or complicated CDI is suspected, initiate empirical treatment as soon as possible. Metronidazole (500 mg tid $\times 10-14$ days) is the drug of choice for the initial episode of mild-moderate CDI. Oral vancomycin (125 mg QID $\times 10-14$ days) is the drug of choice for an initial episode of severe CDI [11]. Oral vancomycin has a comparable rate of cure for patients with CDI, but a lesser chance for recurrence [22] (Table 1).

It is important to recognize that metronidazole has potential adverse risks. Patients given metronidazole may have a disulfiram-like reaction if they consume alcohol while on therapy. There are potential carcinogenic properties to metronidazole as well. Thrombophlebitis has been associated with intravenous administration of metronidazole. Additionally, neurotoxicity (paresthesias, dysarthria, etc.) has been associated with long-term use of metronidazole. Therefore, long-term therapy with metronidazole should be avoided [4••]. Additionally, there was a recent study which demonstrated the risk of death was significantly higher with intravenous metronidazole than with oral metronidazole or oral vancomycin. Thus, oral metronidazole should be given preferentially over the intravenous route whenever possible [21].

Oral vancomycin is currently the mainstay of treatment for severe, complicated CDI. The recommended dose is $125 \mathrm{mg}$ orally four times daily with or without intravenous metronidazole. If the patient has an ileus, vancomycin can be administered per rectum. The current cost for a 15-day supply of oral vancomycin is $\$ 1,990.97$ [5]. Some hospital pharmacists are starting to administer the intravenous formulation of vancomycin orally to reduce hospital costs, but there are currently no studies to support this practice.

Fidaxomicin (Dificid) is a macrocyclic antibiotic with bactericidal properties that received FDA-approval on May 27,2011 . This medication is given twice daily for ten days and the most common side effects are nausea, vomiting, headache, abdominal pain, and diarrhea. A landmark study compared fidaxomicin to vancomycin and found no significant difference in cure rates between the two agents for CDI. However, there was a significant reduction in recurrences between fidaxomicin and vancomycin (15.4 vs.
$25.3 \%$ ). However, the current cost of fidaxomicin is $\$ 2,800$ for a 10 -day course and this might restrict widespread use of this agent for now [15].

\section{Recurrence}

Approximately $25 \%$ of cases of CDI recur. Most recurrences present within 1-3 weeks of discontinuing treatment, but can present as much as 2-3 months later. If a patient has one recurrence, there is a $45-65 \%$ chance of an additional recurrence. For the first recurrence of mildmoderate CDI, metronidazole is the preferred antimicrobial. For each subsequent recurrence, oral vancomycin with a tapering dose is the recommended therapy. In these cases, the patient should receive vancomycin $125 \mathrm{mg}$ orally four times daily for 10-14 days, then $125 \mathrm{mg}$ twice daily for a week, then $125 \mathrm{mg}$ daily for a week, then $125 \mathrm{mg}$ every two or three days for 2-8 weeks [4••].

\section{Infection Control}

Healthcare workers and visitors must use gloves and gowns when examining or visiting a patient with CDI. Hand hygiene with an antimicrobial soap should be used and alcohol-based hand rubs should be avoided. Patients with known or suspected CDI should be admitted or transferred to a private room. Identification of asymptomatic carriers of $C$. difficile is not recommended. Additionally, reusable electric thermometers should be avoided and disposable thermometers should be used for rectal temperature recordings. Rooms occupied by patients with CDI should be cleaned with chlorine-containing agents or other sporicidal agents [4••].

\section{Probiotics}

Probiotics are live, nonpathogenic bacteria capable of colonizing the colonic mucosa. There is currently no evidence of benefit in severe CDI. They may have some role for recurrent mild-moderate disease ( $S$. boulardii), but the evidence is inconclusive. Furthermore, probiotics are not recommended to prevent primary CDI, as there is limited data to support this approach and there is a potential risk of bloodstream infection $[4 \bullet \bullet, 8 \bullet]$.

Table 1 Treatment recommendations based on severity and recurrence

\begin{tabular}{llll}
\hline $\begin{array}{l}\text { Mild-moderate CDI } \\
(1 \text { st episode })\end{array}$ & $\begin{array}{l}\text { Mild-moderate CDI } \\
(1 \mathrm{st} \text { recurrence })\end{array}$ & $\begin{array}{l}\text { 2nd or more recurrence of mild-moderate } \\
\text { CDI }\end{array}$ & Severe CDI \\
\hline $\begin{array}{l}\text { Metronidazole }(500 \mathrm{mg} \\
\text { tid } \times 10-14 \text { days })\end{array}$ & $\begin{array}{c}\text { Metronidazole }(500 \mathrm{mg} \\
\text { tid } \times 10-14 \text { days })\end{array}$ & $\begin{array}{c}\text { Vancomycin }(125 \mathrm{mg} \text { orally four times daily } \\
\text { for } 10-14 \text { days then } 125 \mathrm{mg} \text { twice daily for } \\
\text { a week, then } 125 \mathrm{mg} \text { daily for a week, then }\end{array}$ & $\begin{array}{l}\text { daily for } 10-14 \text { days }) \pm \text { intravenous } \\
\text { metronidazole and consider vancomycin }\end{array}$ \\
& & $125 \mathrm{mg}$ every 2 or 3 days for 2 to 8 weeks $)$ & taper \\
\hline
\end{tabular}




\section{Fecal Bacteriotherapy}

There has been a significant amount of interest recently in the use of fecal bacteriotherapy in patients with recurrent C. difficile. The rationale of this approach is that stool from a healthy donor might restore normal enteric flora in a patient affected with recurrent CDI and prevent recurrence. This is performed by taking stool from a healthy donor and inserting via enema, colonoscope, nasoduodenal or jejunal tube (an insertion route not recommended by these authors due to potential for patient dysgeusia). A success rate up to $87 \%$ of stopping recurrences have been reported in small observational studies. [13, 16]

\section{Conclusions}

Clostridium difficile colitis is an ever present danger and constant risk for all hospitalized patients. Its continued prevalence throughout our hospital communities serves as a constant reminder that we as health care providers must stay abreast of the most current ways to protect, diagnose, and treat our patients. With the rising cost of healthcare it is imperative that we stress a focus on preventing transmission via barrier methods like gowning and gloving as well as consistent and appropriate use of hand washing and sporicidal agents when in contact with a $C$. difficile infected patient. Most importantly, a solid understanding of treatment options which not only includes proper antibiotic utilization but also proper duration of treatment, are cornerstone elements to preventing the severe complications and recurrence in the at risk patient populations that frequent our hospital wards.

Disclosure No potential conflicts of interest relevant to this article were reported.

\section{References}

Papers of particular interest, published recently, have been highlighted as:

- Of importance

•- Of major importance

1. Centers for Disease Control and Prevention. Morbidity and mortality weekly report. Vital signs: preventing Clostridium difficile infection. MMWR Morb Mortal Wkly Rep. 2012;61(09):157-62.
2. Centers for Disease Control and Prevention. Morbidity and mortality weekly report. 2002; 51: RR-16.

3. Ciaran K, LaMont TJ. Clostridium difficile-more difficult than ever. N Engl J Med. 2008;359:1932-40.

4. - Cohen $\mathrm{S}$ et al. Clinical practice guidelines for Clostridium difficile infections in adults: 2010 update by SHEA and IDSA. Infect Control Hosp Epidemiol. 2010; 31(5): 431-55. This is the most recent evidence-based guidelines published by the Infectious Disease Society of America.

5. http://Drugstore.com. Accessed 11 Dec 2011.

6. Flatmark A, et al. Early colectomy in severe ulcerative colitis. Scand J Gastroenterol. 1975;10(4):427-31.

7. Grieco MB, et al. Toxic megacolon complicating crohns colitis. Ann Surg. 1980;191(1):75-80.

8. - Hempel $\mathrm{S}$ et al. Probiotics for prevention and treatment of antibiotic associated diarrhea. JAMA. 2012; 307(18): 1959-69. This article that provides the most recent evidence regarding probiotics and CDI.

9. $\bullet$ Howell $\mathrm{M}$ et al. Latrogenic gastric acid suppression and the risk of nosocomial Clostridium difficile infection. Arch Intern Med. 2010; 170(9): 784-90. This was the landmark trial that demonstrated an association of proton pump inhibitors and nosocomial CDI.

10. Jabbar Umair, et al. Effectiveness of alcohol-based hand rubs for removal of Clostridium difficile spores from hand. Infect Control Hospital Epidemiol. 2010;31(6):565-70.

11. Jarvis WR, et al. National point prevalence of Clostridium difficile in US healthcare facility inpatients, 2009. Am J Infect Control. 2009;37(4):263-70.

12. Laino C. C. diff on rise in kids-and outside hospital. http:// Webmd.com. children's health section. Accessed 17 June 2012.

13. Landy J, et al. Faecal transplantation therapy for gastrointestinal disease. Aliment Pharmacol Ther. 2011;34(4):409-15.

14. Linsky A, et al. Proton pump inhibition and risk for recurrent Clostridium difficile infection. Arch Intern Med. 2010;170(9):772-8.

15. Louie TJ, et al. Fidaxomicin versus vancomycin for Clostridium difficile infection. N Engl J Med. 2011;364:422-31.

16. Mattila E, et al. Fecal transplantation, through colonoscopy, is effective therapy for recurrent Clostridium difficile infection. Gastroenterology. 2012;142:490-6.

17. Sheth S, LaMont T. Toxic megacolon. http://Uptodate.com. Literature review current through May 2012.

18. Trudel JL, et al. Toxic megacolon complicating pseudomembranous enterocolitis. Dis Colon Rectum. 1995;38(10):1033.

19. Wanahita Anna, et al. Clostridium difficile infection in patients with unexplained leukocytosis. Am J Med. 2010;115(7):543-6.

20. Warney M, et al. Toxin production by an emerging strain of Clostridium difficile associated with outbreaks of severe disease in North America and Europe. Lancet. 2005;366(9491):1079-84.

21. Wenisch JM, et al. Prospective observational study comparing three different treatment regimes in patients with Clostridium difficile infection. Antimicrob Agents Chemother. 2012;56:1974.

22. Zar FA, et al. A comparison of vancomycin and metronidazole for treatment of Clostridium difficile-associated diarrhea, stratified by disease severity. Clin Infect Dis. 2007; 45(3):302-7. 